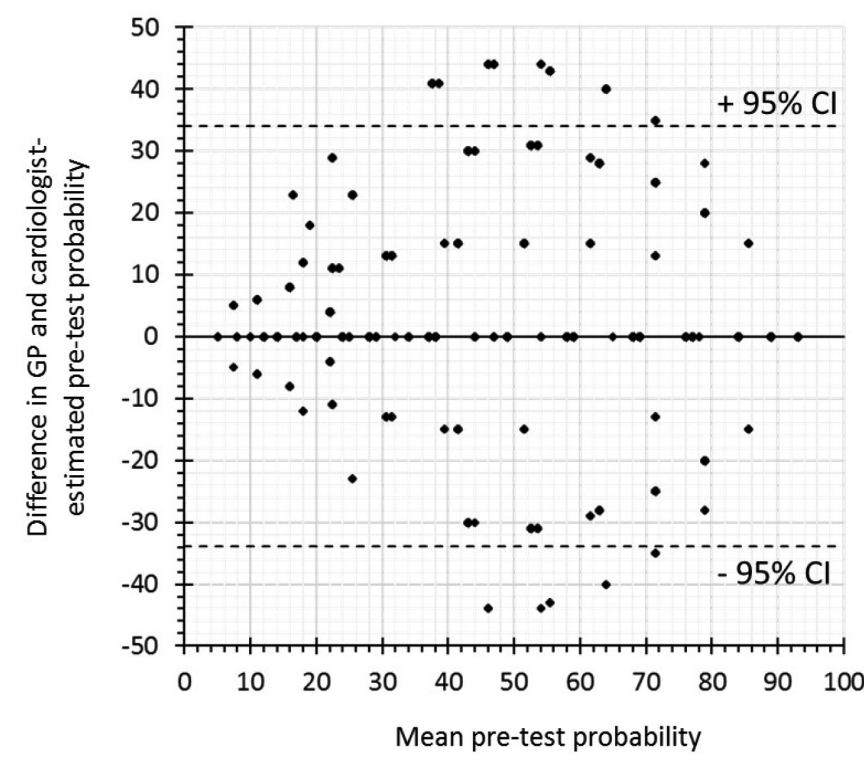

Abstract 82 Figure 1 Bland-Altman plot of agreement between GP and cardiology-estimated pre-test probability for coronary artery disease

to short duration of pain. No data regarding relationship to rest or GTN were available in $n=475$ (29.1\%), attributable to the referrer only considering GTN, which was often only prescribed at the point of referral.

Abstract 82 Table 1 Concordance between GP and RACPC diagnosis of anginal-type chest pain

\begin{tabular}{lllll}
\hline & & \multicolumn{3}{c}{ RACPC diagnosis } \\
\hline \multirow{4}{*}{ GP diagnosis } & Typical angina & Atypical angina & Non-angina \\
\cline { 3 - 5 } & Typical angina & 303 & 140 & 18 \\
& Atypical angina & 324 & 335 & 64 \\
& Non-angina & 141 & 224 & 85 \\
\hline
\end{tabular}

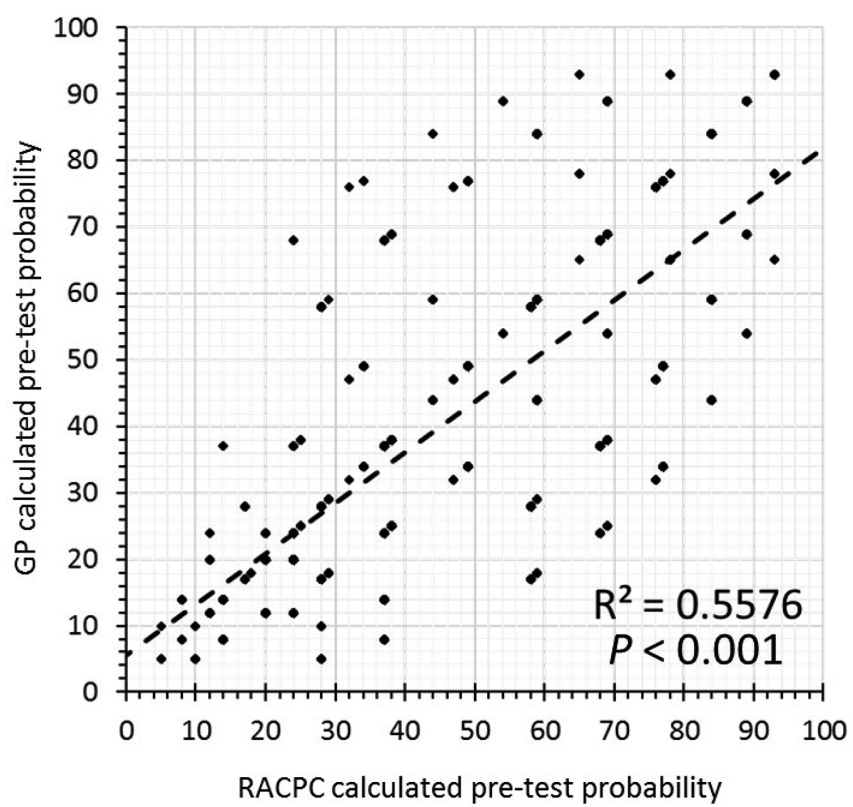

Abstract 82 Figure 2 Correlation between GP and cardiologyestimated pre-test probability for coronary artery disease
Conclusions GP and cardiologist-led diagnosis of stable angina is discordant; the pre-test probability is overestimated in primary care, which would have significant implications for subsequent diagnostic investigation. The RACPC remains an essential service for patients with suspected stable angina.

\section{SELF-ASSESSMENT OF CARDIOVASCULAR RISK: PUBLIC USE OF THE ONLINE JBS3 HEART AGE TOOL}

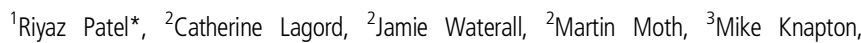
'John Deanfield. ' UCL; ${ }^{2}$ Public Health UK; ${ }^{3}$ British Heart Foundation; * Presenting Author

\subsection{6/heartjnl-2016-309890.83}

Background The JBS3 risk calculator offers a novel approach to cardiovascular risk communication by estimating a "heart age". A modified version of this tool was introduced online to broaden access to personalized risk assessment to the general population and encourage participation in the NHS Health Check programme. This study reports on its early uptake and the profiles of those who used it.

Methods The JBS3 tool estimates a"heart age", through multivariable modelling which is referenced to someone of the same age, gender and ethnicity with optimal risk factors. Between February and July 2015, user data collected from the NHS Choices website (https://www.nhs.uk/tools/pages/heartage. aspx), where the tool was hosted, were analyzed anonymously using standard analytic packages.

Results The online tool was accessed 1.4 million times in the first 5 months, with increased activity following limited media coverage. Of the 575,782 users completing the data journey with a valid"heart age" result, their demographic and risk factor profiles broadly resembled the population of England, although both younger users and males (60\%) were over represented. Almost 50\% and $79 \%$ did not know or enter their blood pressure or cholesterol values, respectively (Figure 1). Estimated"heart age" was higher than chronological age for $79 \%$ of all users, but also for $69 \%$ of younger users under 40 years who are at low 10-year risk and not invited for NHS Health Checks (Figure 2).

Conclusions/interpretation These data suggest a high level of public interest in self-assessment of cardiovascular risk when an easily understood metric is used, although a large number

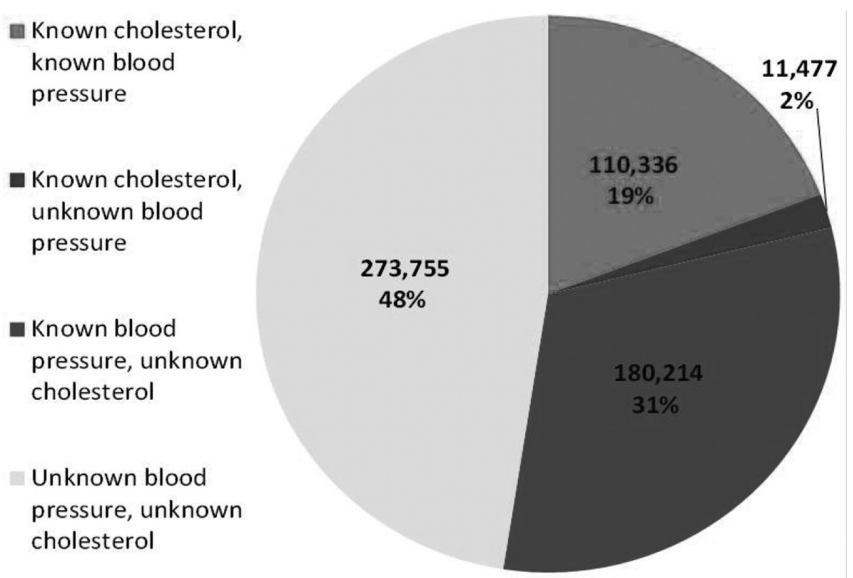

Abstract 83 Figure 1 Proportion of users who were unaware of their blood preasure and/or cholesterol 


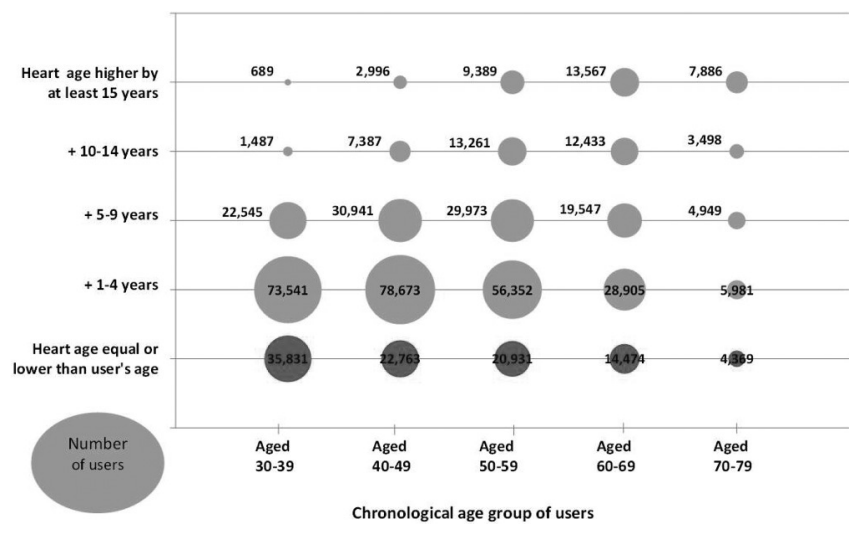

Abstract 83 Figure 2 Heart age estimates with proportions who are older then their heart age, by age group of users

of users lack awareness of their own risk factors. The Heart Age tool was accessed by a group not easily reached by conventional approaches yet is at high cardiovascular risk and would benefit most from early and sustained risk reduction. These are both important opportunities for interventions to educate and empower the public to manage better their cardiovascular risk and promote population level prevention.

\section{CARDIOVASCULAR RISK REDUCTION USING CONTEMPORARY GUIDELINE RECOMMENDATIONS: OUTCOMES OF THE HEART ATTACK PREVENTION PROGRAMME FOR YOU (HAPPY) LONDON STUDY}

Mohammed Khanji*, Armida Balawon, Redha Boubertakh, Steffen Petersen. Centre for Advanced Cardiovascular Imaging and Research, Queen Mary University London; *Presenting Author

\subsection{6/heartjnl-2016-309890.84}

Introduction Cardiovascular disease (CVD) remains the leading cause of morbidity and mortality globally. The importance of primary prevention (PP) of CVD is now well recognised and can potentially improve quality of life and survival costeffectively.

Aim To assess benefits of contemporary guideline based PP recommendations for CVD risk reduction in those with elevated global cardiovascular risk.

Methods 402 participants aged 40-74 years were recruited as part of the HAPPY London study. Eligible participants had an elevated 10-year risk based on a QRISK2 score of $10 \%$ or more. Internet access was required to be able to register and complete questionnaires online. Individualised one-off cardiovascular risk reduction advice was provided, based on the JBS3 and ESC 2012 CVD prevention guidelines. Follow-up at 3 months assessed changes in risk factors and risk scores using paired t-test statistical analysis.

Results Mean age 65 years; 37\% females and 370 (92\%) completed the 3-months follow up. There was a small but significant improvement in mean blood pressure from 132/80 $\mathrm{mmHg}$ to $129 / 78 \mathrm{mmHg}(\mathrm{p}<0.001)$. Other improvements included weight, waist circumference, fasting glucose, total cholesterol, physical activity, Framingham and QRISK2 scores (Table 1).

QRISK2 score dropped from $18.9 \%$ to $18.5 \%$ at followup. This was a $0.7 \%$ absolute reduction compared to expected
Abstract 84 Table 1 Baseline and follow up measurements

\begin{tabular}{llllll}
\hline Variable (mean) & Baseline & 3 Months & P value & $\mathbf{9 5 \%}$ & $\mathbf{C l}$ of differences \\
\hline Systolic BP (mmHg) & 132.4 & 129 & $<0.001$ & 2.36 & 4.77 \\
Diastolic BP (mmHg) & 79.6 & 78 & $<0.001$ & 1.00 & 2.35 \\
Weight (kg) & 80.3 & 79.2 & $<0.001$ & 0.55 & 1.02 \\
BMI (kg/cm2) & 27.7 & 27.4 & $<0.001$ & 0.18 & 0.34 \\
Waist circumference (cm) & 95.6 & 93.8 & $<0.001$ & 0.96 & 1.92 \\
Framingham score (\%) & 17.4 & 15.8 & $<0.001$ & 1.04 & 2.1 \\
QRISK2 score (\%) & 18.9 & 18.5 & 0.016 & 0.06 & 0.57 \\
Total cholesterol & 5.0 & 4.8 & $<0.001$ & 0.17 & 0.31 \\
Glucose & 5.8 & 5.6 & $<0.001$ & 0.1 & 0.3 \\
LDL & 2.8 & 2.7 & $<0.001$ & 0.09 & 0.22 \\
\hline
\end{tabular}

resulting in about 660 less CVD events per 100,000 over 10 years.

Conclusions Implementing guideline recommendation for CVD PP can produce modest improvements in risk factors in the medium term in patients at high cardiovascular risk. Promoting long-term compliance should help lower future cardiovascular events.

\section{SOFT DRINKS AND SWEETENED BEVERAGES AND THE RISK OF CARDIOVASCULAR DISEASE AND MORTALITY A SYSTEMATIC REVIEW AND META-ANALYSIS}

Aditya Narain. Keele University

\subsection{6/heartjnl-2016-309890.85}

Background Despite the well-characterised association between sweetened beverage intake and development of cardio-metabolic risk factors, the relationship between sweetened beverage intake and cardiovascular disease and mortality is controversial. A systematic review and meta-analysis was conducted to evaluate the relationship between sweetened beverages and cardiovascular events and mortality.

Methods Medline and EMBASE were searched in July 2015 for studies that considered soft drink intake and their association with risk of mortality, coronary heart disease (CHD) or stroke. Pooled risk ratios for adverse outcomes were calculated using inverse variance with a random effects model, and heterogeneity was assessed using the $\mathrm{I}^{2}$ statistic.

Results 8 studies with 308,810 participants (34-75 years) were included in the review. Pooled results suggest a significant increase in stroke RR 1.13 95\% CI 1.02-1.24, and CHD RR $1.2295 \%$ CI 1.14-1.30 with incremental increase in sugarsweetened beverage (SSB) consumption. For stroke and CHD, there were significant increases with greater incremental increase in artificially sweetened beverage (ASB) consumption (stroke RR 1.13 95\% CI 1.02-1.24, CHD RR 1.22 95\% CI 1.14-1.30), but not vascular events. For high versus low SSB, there was a significant increase in CHD (RR 1.19 95\% CI 1.09-1.31) but not stroke, vascular events or mortality. For ASB, there was a significant increase in stroke (RR 1.14 95\% CI 1.04-1.26) and vascular events (RR 1.44 95\% CI 1.02-2.03) but not CHD.

Conclusions Current evidence suggests an association between increased cardiovascular risk and greater consumption of sugar-sweetened beverages, although consumption may be a surrogate for adverse health behaviours. 